\title{
Frequency Weighted Model Order Reduction Technique and Error Bounds for Discrete Time Systems
}

\author{
Muhammad Imran, ${ }^{1}$ Abdul Ghafoor, ${ }^{1}$ and Victor Sreeram ${ }^{2}$ \\ ${ }^{1}$ Military College of Signals, National University of Sciences and Technology (NUST), Islamabad, Pakistan \\ ${ }^{2}$ School of Electrical, Electronic and Computer Engineering, University of Western Australia, Crawley, WA 6009, Australia \\ Correspondence should be addressed to Abdul Ghafoor; abdulghafoor-mcs@nust.edu.pk
}

Received 15 December 2013; Revised 10 February 2014; Accepted 11 February 2014; Published 18 March 2014

Academic Editor: Xiaojie Su

Copyright (C) 2014 Muhammad Imran et al. This is an open access article distributed under the Creative Commons Attribution License, which permits unrestricted use, distribution, and reproduction in any medium, provided the original work is properly cited.

\begin{abstract}
Model reduction is a process of approximating higher order original models by comparatively lower order models with reasonable accuracy in order to provide ease in design, modeling and simulation for large complex systems. Generally, model reduction techniques approximate the higher order systems for whole frequency range. However, certain applications (like controller reduction) require frequency weighted approximation, which introduce the concept of using frequency weights in model reduction techniques. Limitations of some existing frequency weighted model reduction techniques include lack of stability of reduced order models (for two sided weighting case) and frequency response error bounds. A new frequency weighted technique for balanced model reduction for discrete time systems is proposed. The proposed technique guarantees stable reduced order models even for the case when two sided weightings are present. Efficient technique for frequency weighted Gramians is also proposed. Results are compared with other existing frequency weighted model reduction techniques for discrete time systems. Moreover, the proposed technique yields frequency response error bounds.
\end{abstract}

\section{Introduction}

Model reduction has played a significant role in modern control system design and caught a lot of attention in the last few decades [1-5]. It is desirable that reduced order model preserves the fundamental properties of original system like stability, passivity, and so forth. Moreover, the approximation error between original and reduced order system is required to be small. Balanced truncation [6] is prominent model reduction scheme, which not only ensures stability of reduced order systems but also provides frequency response a priori error bounds. Other schemes like Hankel norm approximation [7], Pade approximation [8], Krylov technique $[9,10]$, linear matrix inequality (LMI) technique $[11,12]$, and so forth, are also useful for model reduction. Hankel norm approximation has complex implementation and does not preserve steady state [13]. Pade and Krylov approximation sometimes provide unstable reduced order models and there exist no global error bounds [14, 15]. LMI technique is based on mathematical iterative methods (i.e., convex optimization and bisection algorithm); therefore, much computational power is consumed [16]. However, LMI technique has been applied on various model reduction problems for different types of systems (including time delay [17], discrete state delay [18, 19], switched hybrid [20], and nonlinear stochastic [21]). Applications of model reduction are not only restricted to control engineering but also find utility in medical [22] and text summarization [23] areas, and so forth. Most model reduction algorithms tend to minimize the reduction error over the whole frequency range; however there are certain situations (like controller reduction [24]), wherein the approximation error is more critical over a certain band of frequencies.

Enns [25] has enhanced the balanced truncation [6] to incorporate frequency weights for model reduction of higher order systems. This technique can work with input, output, and two sided weightings. However, in two sided weighting case, this technique may give unstable reduced order models [26]. To circumvent instability issue in the presence of two sided weightings, many techniques appear in the literature (including Lin and Chiu [27], Wang et al. [28], Varga and Anderson [29], Ghafoor and Sreeram [30], etc.). 
In Lin and Chiu [27] technique weightings are strictly proper. It was improved to include more general proper weightings in [26]. Varga and Anderson [29] technique yields proper model for strictly proper systems also. Ghafoor and Sreeram [30] technique is a parameterized technique. Wang et al's technique is relatively useful, since other techniques $[27,29]$ are not applicable for controller order reduction. Moreover, Wang et al's technique yields easily computable expression for a priori error bounds.

Various partial fraction based techniques appear in the literature [31-35] that works for continuous as well as discrete time systems. Unfortunately, most of these techniques yield large frequency response error [30] as compared to Enns technique. However, [35] incorporates free parameters to reduce the approximation error.

Most frequently, frequency weighted model reduction problem is treated in continuous time; however, there are few papers (like [36-38]) which deal with this problem explicitly in discrete time. Sahlan et al.s [36] technique (discrete time version of [31]) is a modified version of Lin and Chiu [27] technique. Campbell et al.s [37] technique is discrete time counterpart of Wang et al.s [28] technique, which not only provides stability for two sided case but also gives easily computable error bounds. Campbell et al.'s [37] technique involves taking absolute values of eigenvalues. This may introduce a large change to the system and hence a larger error if it contains some negative eigenvalues. Varga and Anderson's [38] tend to minimize the distance between Enns and Campbell et al's techniques Gramians by eliminating negative eigenvalues.

In this work, a technique is developed for frequency weighted balanced model order reduction for discrete time systems. The large change in eigenvalues is avoided by yielding similar effect on all eigenvalues. The stability is guaranteed even for double sided weighting. The proposed technique provides comparable frequency response error and yields easily computable a priori error bounds. Numerical examples are given to show the usefulness and comparison of proposed technique with the existing frequency weighted balanced reduction techniques.

\section{Preliminaries}

In this section we review some of the existing frequency weighted model reduction techniques for discrete time systems which include Enns [25], Campbell et al.s [37], and Varga and Anderson's [38].

Consider a stable full order original system with transfer function $H(z)=C(z I-A)^{-1} B+D$, a stable input weighting system with input transfer function $V_{i}(z)=C_{i}\left(z I-A_{i}\right)^{-1} B_{i}+$ $D_{i}$, and a stable output weighting system with output transfer function $W_{o}(z)=C_{o}\left(z I-A_{o}\right)^{-1} B_{o}+D_{o}$; the augmented systems are given by

$$
\begin{gathered}
H(z) V_{i}(z)=C_{d i}\left(z I-A_{d i}\right)^{-1} B_{d i}+D_{d i}, \\
W_{o}(z) H(z)=C_{d o}\left(z I-A_{d o}\right)^{-1} B_{d o}+D_{d o},
\end{gathered}
$$

where

$$
\begin{gathered}
{\left[\begin{array}{c|c}
A_{d i} & B_{d i} \\
\hline C_{d i} & D_{d i}
\end{array}\right]=\left[\begin{array}{cc|c}
A & B C_{i} & B D_{i} \\
0 & A_{i} & B_{i} \\
\hline C & D C_{i} & D D_{i}
\end{array}\right],} \\
{\left[\begin{array}{c|c}
A_{d o} & B_{d o} \\
\hline C_{d o} & D_{d o}
\end{array}\right]=\left[\begin{array}{cc|c}
A_{o} & B_{o} C & B_{o} D \\
0 & A & B \\
\hline C_{o} & D_{o} C & D_{o} D
\end{array}\right] .}
\end{gathered}
$$

Let the Gramians

$$
P_{d i}=\left[\begin{array}{cc}
P_{e n} & P_{12} \\
P_{12}^{T} & P_{V}
\end{array}\right], \quad Q_{d o}=\left[\begin{array}{ll}
Q_{W} & Q_{12}^{T} \\
Q_{12} & Q_{e n}
\end{array}\right]
$$

satisfy the following Lyapunov equations:

$$
\begin{gathered}
A_{d i} P_{d i} A_{d i}^{T}-P_{d i}+B_{d i} B_{d i}^{T}=0, \\
A_{d o}^{T} Q_{d o} A_{d o}-Q_{d o}+C_{d o}^{T} C_{d o}=0 .
\end{gathered}
$$

Expanding the $(1,1)$ and $(2,2)$ blocks of above equations, we get

$$
\begin{aligned}
& A P_{e n} A^{T}-P_{e n}+X_{e n}=0, \\
& A^{T} Q_{e n} A-Q_{e n}+Y_{e n}=0,
\end{aligned}
$$

where

$$
\begin{aligned}
X_{e n}=A P_{12} C_{i}^{T} B^{T} & +B C_{i} P_{12}^{T} A^{T}+B C_{i} P_{V} C_{i}^{T} B^{T}+B D_{i} D_{i}^{T} B^{T}, \\
Y_{e n}= & C^{T} B_{o}^{T} Q_{12}^{T} A+A^{T} Q_{12} B_{o} C \\
& +C^{T} B_{o}^{T} Q_{W} B_{o} C+C^{T} D_{o}^{T} D_{o} C .
\end{aligned}
$$

2.1. Enns Technique [25]. Let $T$ be contragredient matrix obtained as

$$
T^{T} Q_{e n} T=T^{-1} P_{e n} T^{-T}=\operatorname{diag}\left\{\sigma_{1}, \sigma_{2}, \sigma_{3}, \ldots, \sigma_{n}\right\},
$$

where $\sigma_{j} \geq \sigma_{j+1}, j=1,2,3, \ldots, n-1$ and $\sigma_{l}>\sigma_{l+1}$. By transforming and then partitioning the original system, we have

$$
\begin{gathered}
\widehat{A}=T^{-1} A T=\left[\begin{array}{ll}
A_{11} & A_{12} \\
A_{21} & A_{22}
\end{array}\right], \quad \widehat{B}=T^{-1} B=\left[\begin{array}{l}
B_{1} \\
B_{2}
\end{array}\right], \\
\widehat{C}=C T=\left[\begin{array}{ll}
C_{1} & C_{2}
\end{array}\right], \quad \widehat{D}=D,
\end{gathered}
$$

where $A_{11} \in R^{l \times l}$. The reduced order system is obtained as follows: $H_{l}(z)=C_{1}\left(z I-A_{11}\right)^{-1} B_{1}+D$.

Remark 1. It is not guaranteed to ensure that $X_{\text {en }} \geq 0$ and $Y_{\text {en }} \geq 0$; the reduced order models obtained using Enns technique may not remain stable for both sided weightings. 
2.2. Campbell et al.'s Technique [37]. Campbell et al.s [37] (a discrete time version of [28]) guarantees the positive semidefiniteness of symmetric matrices $X_{e n}$ and $Y_{e n}$ to ensure stability. Let the new controllability $P_{C S}$ and observability $Q_{C S}$ Gramians, respectively, be calculated by solving the following Lyapunov equations:

$$
\begin{aligned}
& A P_{C S} A^{T}-P_{C S}+B_{C S} B_{C S}^{T}=0, \\
& A^{T} Q_{C S} A+Q_{C S}+C_{C S}^{T} C_{C S}=0 .
\end{aligned}
$$

which are used to obtain contragredient matrix $T$ as

$$
T^{T} Q_{C S} T=T^{-1} P_{C S} T^{-T}=\Sigma,
$$

where $\Sigma=\operatorname{diag}\left\{\sigma_{1}, \sigma_{2}, \sigma_{3}, \ldots, \sigma_{n}\right\}$ and $\sigma_{j} \geq \sigma_{j+1}, j=$ $1,2,3, \ldots, n-1$ and $\sigma_{l}>\sigma_{l+1}$. The fictitious input $B_{C S}$ and output $C_{C S}$ matrices shown in the above Lyapunov equations are defined as $B_{C S}=U_{C S}\left|S_{C S}\right|^{1 / 2}$ and $C_{C S}=$ $\left|R_{C S}\right|^{1 / 2} V_{C S}^{T}$, respectively. Since the expressions $U_{C S}, S_{C S}, V_{C S}$, and $R_{C S}$ are calculated by orthogonal eigen decomposition $X_{\text {en }}=U_{C S} S_{C S} U_{C S}^{T}$ and $Y_{e n}=V_{C S} R_{C S} V_{C S}^{T}$, where $S_{C S}=$ $\operatorname{diag}\left(s_{1}, s_{2}, \ldots, s_{n}\right), R_{C S}=\operatorname{diag}\left(r_{1}, r_{2}, \ldots, r_{n}\right),\left|s_{1}\right| \geq\left|s_{2}\right| \geq$ $\cdots \geq\left|s_{n}\right| \geq 0$ and $\left|r_{1}\right| \geq\left|r_{2}\right| \geq \cdots \geq\left|r_{n}\right| \geq 0$. The reduced order systems are calculated by transforming and then partitioning the original system.

Remark 2. The stability of reduced order models in the presence of both input and output weightings is guaranteed and the following error bound holds [37]

$$
\begin{aligned}
& \left\|W_{o}(z)\left(H(z)-H_{l}(z)\right) V_{i}(z)\right\|_{\infty} \\
& \quad \leq 2\left\|W_{o}(z) L_{C S}\right\|_{\infty}\left\|K_{C S} V_{i}(z)\right\|_{\infty} \sum_{j=l+1}^{n} \sigma_{j},
\end{aligned}
$$

where

$$
\begin{gathered}
L_{C S}=C V_{C S} \operatorname{diag}\left(\left|r_{1}\right|^{-1 / 2},\left|r_{2}\right|^{-1 / 2}, \ldots,\left|r_{l i}\right|^{-1 / 2}, 0, \ldots, 0\right), \\
K_{C S}=\operatorname{diag}\left(\left|s_{1}\right|^{-1 / 2},\left|s_{2}\right|^{-1 / 2}, \ldots,\left|s_{k o}\right|^{-1 / 2}, 0, \ldots, 0\right) U_{C S}^{T} B .
\end{gathered}
$$

$l i=\operatorname{rank}\left[X_{e n}\right]$ and $k o=\operatorname{rank}\left[Y_{e n}\right]$.

2.3. Varga and Anderson's Technique [38]. Note that the Gramians satisfy $P_{e n} \leq P_{C S}$ and $Q_{e n} \leq Q_{C S}$. For minimizing the distances between the Gramians, $P_{e n}-P_{C S}$ and $Q_{e n}-Q_{C S}$, Varga and Anderson proposed the following technique.

Let new controllability and observability Gramians $P_{V d}$ and $Q_{V d}$, respectively, be calculated as the solutions to Lyapunov equations

$$
\begin{aligned}
& A P_{V d} A^{T}-P_{V d}+B_{V d} B_{V d}^{T}=0, \\
& A^{T} Q_{V d} A-Q_{V d}+C_{V d}^{T} C_{V d}=0
\end{aligned}
$$

which are used obtain contragredient matrix $T$ as

$$
T^{T} Q_{V d} T=T^{-1} P_{V d} T^{-T}=\Sigma,
$$

where $\Sigma=\operatorname{diag}\left\{\sigma_{1}, \sigma_{2}, \sigma_{3}, \ldots, \sigma_{n}\right\}$, and $\sigma_{j} \geq \sigma_{j+1}, j=$ $1,2, \ldots, n-1, \sigma_{l}>\sigma_{l+1}$. The new fictitious input $B_{V d}$ and output $C_{V d}$ matrices in the above Lyapunov equations are defined as $B_{V d}=U_{V d_{1}} S_{V d_{1}}^{1 / 2}$ and $C_{V d}=R_{V d_{1}}^{1 / 2} V_{V d_{1}}^{T}$, respectively. The terms $U_{V d_{1}}, S_{V d_{1}}, V_{V d_{1}}$, and $R_{V d_{1}}$ are calculated from the orthogonal eigen decomposition of symmetric matrices

$$
\begin{aligned}
& X_{e n}=\left[\begin{array}{ll}
U_{V d_{1}} & U_{V d_{2}}
\end{array}\right]\left[\begin{array}{cc}
S_{V d_{1}} & 0 \\
0 & S_{V d_{2}}
\end{array}\right]\left[\begin{array}{c}
U_{V d_{1}}^{T} \\
U_{V d_{2}}^{T}
\end{array}\right], \\
& Y_{e n}=\left[\begin{array}{ll}
V_{V d_{1}} & V_{V d_{2}}
\end{array}\right]\left[\begin{array}{cc}
R_{V d_{1}} & 0 \\
0 & R_{V d_{2}}
\end{array}\right]\left[\begin{array}{c}
V_{V d_{1}}^{T} \\
V_{V d_{2}}^{T}
\end{array}\right],
\end{aligned}
$$

where $\left[\begin{array}{cc}S_{V d_{1}} & 0 \\ 0 & S_{V d_{2}}\end{array}\right]=\operatorname{diag}\left\{s_{1}, s_{2}, \ldots, s_{n}\right\},\left[\begin{array}{cc}R_{V d_{1}} & 0 \\ 0 & R_{V d_{2}}\end{array}\right]=$ $\operatorname{diag}\left\{r_{1}, r_{2}, r_{3}, \ldots, r_{n}\right\}, s_{1} \geq s_{2} \geq s_{3} \geq \cdots \geq s_{n}, r_{1} \geq$ $r_{2} \geq r_{3} \geq \cdots \geq r_{n}, S_{V d_{1}}=\operatorname{diag}\left\{s_{1}, s_{2}, s_{3}, \ldots, s_{k}\right\}, R_{V d_{1}}=$ $\operatorname{diag}\left\{r_{1}, r_{2}, r_{3}, \ldots, r_{k}\right\}, s_{1} \geq s_{2} \geq s_{3} \geq \cdots \geq s_{k} \geq 0, r_{1} \geq$ $r_{2} \geq r_{3} \geq \cdots \geq r_{k} \geq 0$.

The reduced order systems are calculated by transforming and then partitioning the original system.

Remark 3. The stability of the reduced system is guaranteed and the following error bound holds [38]

$$
\begin{aligned}
& \left\|W_{o}(z)\left(H(z)-H_{l}(z)\right) V_{i}(z)\right\|_{\infty} \\
& \quad \leq 2\left\|W_{o}(z) L_{V d}\right\|_{\infty}\left\|K_{V d} V_{i}(z)\right\|_{\infty} \sum_{j=l+1}^{n} \sigma_{j},
\end{aligned}
$$

where $L_{V d}=C V_{V d_{1}} R_{V d_{1}}^{-1 / 2}$ and $K_{V d}=S_{V d_{1}}^{-1 / 2} U_{V d_{1}}^{T} B$.

\section{Main Results}

In Campbell et al.s [37] technique, the symmetric matrices $X_{e n}$ and $Y_{e n}$ are guaranteed positive/semipositive definite by taking the square root of absolute values of the eigenvalues obtained by eigen decomposition of symmetric matrices $X_{e n}$ and $Y_{e n}$. This may lead to a large change in some eigenvalues and may not affect other eigenvalues. Although in Varga and Anderson's [38] technique, this large change was slightly improved by eliminating negative eigenvalues, but the problem persists with the other eigenvalues. In the following, a new technique is proposed in which a similar effect on all eigenvalues of indefinite matrices $X_{e n}$ and $Y_{e n}$ guarantees stability, error bound, and improved frequency response error.

3.1. Proposed Technique. Let a new controllability $P_{I G}$ and observability $Q_{I G}$ Gramians, respectively, be calculated by solving the following Lyapunov equations:

$$
\begin{aligned}
& A P_{I G} A^{T}-P_{I G}+B_{I G} B_{I G}^{T}=0, \\
& A^{T} Q_{I G} A-Q_{I G}+C_{I G}^{T} C_{I G}=0 .
\end{aligned}
$$


The matrices $B_{I G}$ and $C_{I G}$ are the new fictitious input and output matrices, respectively, and are defined as

$$
\begin{gathered}
B_{I G}= \begin{cases}U_{I G}\left(S_{I G}-s_{n} I\right)^{1 / 2} & \text { for } s_{n}<0 \\
U_{I G} S_{I G}^{1 / 2} & \text { for } s_{n} \geq 0,\end{cases} \\
C_{I G}= \begin{cases}\left(R_{I G}-r_{n} I\right)^{1 / 2} V_{I G}^{T} & \text { for } r_{n}<0 \\
R_{I G}^{1 / 2} V_{I G}^{T} & \text { for } r_{n} \geq 0 .\end{cases}
\end{gathered}
$$

The terms $U_{I G}, S_{I G}, V_{I G}$, and $R_{I G}$ are calculated by the orthogonal eigen decomposition of symmetric matrices $X_{e n}=U_{I G} S_{I G} U_{I G}^{T}$ and $Y_{\text {en }}=V_{I G} R_{I G} V_{I G}^{T}$, where $S_{I G}=$ $\operatorname{diag}\left(s_{1}, s_{2}, s_{3}, \ldots, s_{n}\right), R_{I G}=\operatorname{diag}\left(r_{1}, r_{2}, r_{3}, \ldots, r_{n}\right), s_{1} \geq s_{2} \geq$ $\cdots \geq s_{n}$, and $r_{1} \geq r_{2} \geq \cdots \geq r_{n}$.

Let a contragradient transformation matrix $T$ be obtained as

$$
T^{T} Q_{I G} T=T^{-1} P_{I G} T^{-T}=\Sigma,
$$

where $\Sigma=\operatorname{diag}\left\{\sigma_{1}, \sigma_{2}, \sigma_{3}, \ldots, \sigma_{n}\right\}$, and $\sigma_{j} \geq \sigma_{j+1}, j=$ $1,2, \ldots, n-1, \sigma_{l}>\sigma_{l+1}$. The reduced order system is calculated by transforming and partitioning the original system.

Remark 4. Since $X_{e n} \leq B_{I G} B_{I G}^{T} \geq 0, Y_{\text {en }} \leq C_{I G}^{T} C_{I G} \geq 0$, $P_{I G}>0$ and $Q_{I G}>0$, therefore, the realization $\left(A, B_{I G S}, C_{I G S}\right)$ is minimal. Moreover, the reduced order models are stable.

Theorem 5. The following error bound for the proposed technique holds if the rank conditions $\operatorname{rank}\left[\begin{array}{ll}B_{I G} & B\end{array}\right]=\operatorname{rank}\left[B_{I G}\right]$ and $\operatorname{rank}\left[\begin{array}{c}C_{I G} \\ C\end{array}\right]=\operatorname{rank}\left[C_{I G}\right]$ (which follows from $[2,28,29$, 38]) are satisfied

(i) $\left\|W_{o}(z)\left(H(z)-H_{l}(z)\right) V_{i}(z)\right\|_{\infty}$

$$
\leq 2\left\|W_{o}(z) L_{I G}\right\|_{\infty}\left\|K_{I G} V_{i}(z)\right\|_{\infty} \sum_{j=l+1}^{n} \sigma_{j}
$$

(ii) $\left\|\left(H(z)-H_{l}(z)\right) V_{i}(z)\right\|_{\infty} \leq 2\left\|K_{I G} V_{i}(z)\right\|_{\infty} \sum_{j=l+1}^{n} \sigma_{j}$

(iii) $\left\|W_{o}(z)\left(H(z)-H_{l}(z)\right)\right\|_{\infty}$

$$
\leq 2\left\|W_{o}(z) L_{I G}\right\|_{\infty} \sum_{j=l+1}^{n} \sigma_{j},
$$

where

$$
\begin{aligned}
L_{I G} & = \begin{cases}C V\left(R_{I G}-r_{n} I\right)^{-1 / 2} & \text { for } r_{n}<0 \\
C V R_{I G}^{-1 / 2} & \text { for } r_{n} \geq 0,\end{cases} \\
K_{I G} & = \begin{cases}\left(S_{I G}-s_{n} I\right)^{-1 / 2} U^{T} B & \text { for } s_{n}<0 \\
S_{I G}^{-1 / 2} U^{T} B & \text { for } s_{n} \geq 0 .\end{cases}
\end{aligned}
$$

Proof. We show proof of (i) (whereas (ii) and (iii) are special cases of (i)). Since the rank conditions $\operatorname{rank}\left[\begin{array}{ll}B_{I G} & B\end{array}\right]=$ $\operatorname{rank}\left[B_{I G}\right]$ and $\operatorname{rank}\left[\begin{array}{c}C_{I G} \\ C\end{array}\right]=\operatorname{rank}\left[C_{I G}\right]$ are satisfied, the relationships $B=B_{I G} K_{I G}$ and $C=L_{I G} C_{I G}$ hold. By partitioning $B_{I G}=\left[\begin{array}{l}B_{I G_{1}} \\ B_{I G_{2}}\end{array}\right], C_{I G}=\left[\begin{array}{ll}C_{I G_{1}} & C_{I G_{2}}\end{array}\right]$ and then substituting $B_{1}=B_{I G_{1}} K_{I G}, C_{1}=L_{I G} C_{I G_{1}}$, respectively, yields

$$
\begin{aligned}
& \left\|W_{o}(z)\left(H(z)-H_{l}(z)\right) V_{i}(z)\right\|_{\infty} \\
& =\left\|W_{o}(z)\left(C(z I-A)^{-1} B-C_{1}\left(z I-A_{11}\right)^{-1} B_{1}\right) V_{i}(z)\right\|_{\infty} \\
& =\| W_{o}(z)\left(L_{I G} C_{I G}(z I-A)^{-1} B_{I G} K_{I G}\right. \\
& \left.\quad-L_{I G} C_{I G_{1}}\left(z I-A_{11}\right)^{-1} B_{I G_{1}} K_{I G}\right) V_{i}(z) \|_{\infty} \\
& =\| W_{o}(z) L_{I G}\left(C_{I G}(z I-A)^{-1} B_{I G}\right. \\
& \left.\quad-C_{I G_{1}}\left(z I-A_{11}\right)^{-1} B_{I G_{1}}\right) K_{I G} V_{i}(z) \|_{\infty} \\
& =\left\|W_{o}(z) L_{I G}\right\|_{\infty} \\
& \quad \times\left\|\left(C_{I G}(z I-A)^{-1} B_{I G}-C_{I G_{1}}\left(z I-A_{11}\right)^{-1} B_{I G_{1}}\right)\right\|_{\infty} \\
& \quad \times\left\|K_{I G} V_{i}(z)\right\|_{\infty} .
\end{aligned}
$$

If $\left\{A_{11}, B_{I G_{1}}, C_{I G_{1}}\right\}$ is reduced order model obtained by partitioning a balanced realization $\left\{A, B_{I G}, C_{I G}\right\}$, we have $[7,39]$

$$
\left\|\left(C_{I G}(z I-A)^{-1} B_{I G}-C_{I G_{1}}\left(z I-A_{11}\right)^{-1} B_{I G_{1}}\right)\right\|_{\infty} \leq 2 \sum_{j=l+1}^{n} \sigma_{j} .
$$

Therefore,

$$
\begin{aligned}
& \left\|W_{o}(z)\left(H(z)-H_{l}(z)\right) V_{i}(z)\right\|_{\infty} \\
& \leq 2\left\|W_{o}(z) L_{I G}\right\|_{\infty}\left\|K_{I G} V_{i}(z)\right\|_{\infty} \sum_{j=l+1}^{n} \sigma_{j} .
\end{aligned}
$$

Remark 6. For the case when symmetric matrices $X_{e n} \geq$ 0 and $Y_{e n} \geq 0$, then $P_{e n}=P_{C S}=P_{V d}=P_{I G}$ and $Q_{e n}=Q_{C S}=Q_{V d}=Q_{I G}$. However, when matrices $X_{e n}$ and $Y_{e n}$ are indefinite, then $P_{e n}<P_{I G}$ and $Q_{e n}<Q_{I G}$. Moreover, frequency weighted Hankel singular values satisfy $\left(\lambda_{j}\left[P_{e n} Q_{e n}\right]\right)^{1 / 2} \leq\left(\lambda_{j}\left[P_{I G} Q_{I G}\right]\right)^{1 / 2}$.

Remark 7. For the case when input $V_{i}(z)$ weights are co-inner and output $W_{o}(z)$ weights are inner [40], then $P=P_{\text {en }}=$ $P_{C S}=P_{V d}=P_{I G}$ and $Q=Q_{e n}=Q_{C S}=Q_{V d}=Q_{I G}$, where $P$ and $Q$ are unweighted Gramians defined as

$$
\begin{aligned}
& A P A^{T}-P+B B^{T}=0, \\
& A^{T} Q A-Q+C^{T} C=0 .
\end{aligned}
$$

Remark 8. For the case when the symmetric matrices $X_{e n} \geq 0$ and $Y_{e n} \geq 0$, the reduced order models obtained using Enns [25], Campbell et al.s [37], Varga and Anderson's [38], and proposed technique are same. 
3.2. Computational Aspects. The frequency weighted balanced truncation model reduction techniques balance the original system and then truncate the balanced realization to get the desired reduced order system. The balancing procedure involves computation of transformation matrix from controllability and observability Gramians. Sometimes these matrices become numerically low rank especially in large scale systems (possibly) due to rapid decay of their eigenvalues [3]. Due to this reason, balancing procedure becomes inefficient. Accuracy enhancing techniques for different frequency weighted model reduction techniques appear in $[4,29]$.

For unweighted case, Hammarling's technique [41] is used to obtained Cholesky factors of Gramian matrices from original system realization without actually computing controllability and observability Gramian matrices, respectively.

In frequency weighted techniques, Cholesky factors of the Gramian matrices are obtained from the augmented system realizations. Let $\bar{S}$ and $\bar{R}$ be the Cholesky factors of the augmented system Gramians matrices $P_{b i}$ and $Q_{b o}$ of (4) and (5), respectively,

$$
\begin{aligned}
P_{b i} & =\bar{S} \bar{S}^{T}=\left[\begin{array}{cc}
S_{11} & S_{12} \\
0 & S_{22}
\end{array}\right]\left[\begin{array}{cc}
S_{11}^{T} & 0 \\
S_{12}^{T} & S_{22}^{T}
\end{array}\right] \\
& =\left[\begin{array}{cc}
S_{11} S_{11}^{T}+S_{12} S_{12}^{T} & S_{12} S_{22}^{T} \\
S_{22} S_{12}^{T} & S_{22} S_{22}^{T}
\end{array}\right]=\left[\begin{array}{cc}
P_{\text {en }} & P_{12} \\
P_{12}^{T} & P_{V}
\end{array}\right], \\
Q_{b o} & =\bar{R}^{T} \bar{R}=\left[\begin{array}{cc}
R_{11}^{T} & 0 \\
R_{12}^{T} & R_{22}^{T}
\end{array}\right]\left[\begin{array}{cc}
R_{11} & R_{12} \\
0 & R_{22}
\end{array}\right] \\
& =\left[\begin{array}{ll}
R_{11}^{T} R_{11} & R_{11}^{T} R_{12} \\
R_{12}^{T} R_{11} & R_{22}^{T} R_{22}+R_{12}^{T} R_{12}
\end{array}\right]=\left[\begin{array}{ll}
Q_{W} & Q_{12}^{T} \\
Q_{12} & Q_{e n}
\end{array}\right] .
\end{aligned}
$$

By making use of Cholesky factors $\bar{S}$ and $\bar{R}$ calculated above, the Cholesky factors corresponding to Gramians in frequency weighted model reduction techniques like Enns [25], Campbell et al.s [37], Varga and Anderson's [38], and proposed technique can be obtained as follows:

(1) Enns Technique. The Cholesky factors $S_{e n}=\left[\begin{array}{ll}S_{11} & S_{12}\end{array}\right]$ and $R_{e n}=\left[\begin{array}{l}R_{12} \\ R_{22}\end{array}\right]$ satisfy [29]

$$
\begin{gathered}
P_{e n}=S_{e n} S_{e n}^{T}=S_{11} S_{11}^{T}+S_{12} S_{12}^{T}=\left[\begin{array}{ll}
S_{11} & S_{12}
\end{array}\right]\left[\begin{array}{c}
S_{11}^{T} \\
S_{22}^{T}
\end{array}\right], \\
Q_{e n}=R_{e n}^{T} R_{e n}=R_{22}^{T} R_{22}+R_{12}^{T} R_{12}=\left[\begin{array}{ll}
R_{22}^{T} & R_{12}^{T}
\end{array}\right]\left[\begin{array}{l}
R_{22} \\
R_{12}
\end{array}\right] .
\end{gathered}
$$

(2) Campbell et al.'s Technique. The Cholesky factors $\bar{S}_{C S}$ and $\bar{R}_{C S}$ satisfy $P_{C S}=\bar{S}_{C S} \bar{S}_{C S}^{T}$ and $Q_{C S}=\bar{R}_{C S}^{T} \bar{R}_{C S}[4]$.

(3) Varga and Anderson's Technique. The Cholesky factors $\bar{S}_{V d}$ and $\bar{R}_{V d}$ satisfy $P_{V d}=\bar{S}_{V d} \bar{S}_{V d}^{T}$ and $Q_{V d}=\bar{R}_{V d}^{T} \bar{R}_{V d}$ [4].

(4) Proposed Technique. The Cholesky factors $\bar{S}_{I G}$ and $\bar{R}_{I G}$ satisfy $P_{I G}=\bar{S}_{I G} \bar{S}_{I G}^{T}$ and $Q_{I G}=\bar{R}_{I G}^{T} \bar{R}_{I G}$.

In the following we establish a relationship between Cholesky factors of Gramian matrices used in Enns and proposed techniques. Equations (18) and (19) can be expressed as

$$
\begin{aligned}
& A\left(P_{e n}+P_{a d}\right) A^{T}-\left(P_{e n}+P_{a d}\right) \\
& +\left(X_{e n}-s_{n} I\right)=0, \quad \text { for } s_{n}<0, \\
& A P_{e n} A^{T}-P_{e n}+X_{e n}=0, \quad \text { for } s_{n} \geq 0 \text {, } \\
& A^{T}\left(Q_{e n}+Q_{a d}\right) A-\left(Q_{e n}+Q_{a d}\right) \\
& +\left(Y_{e n}-r_{n} I\right)=0, \quad \text { for } r_{n}<0, \\
& A^{T} Q_{e n} A-Q_{e n}+Y_{e n}=0, \quad \text { for } r_{n} \geq 0 \text {, } \\
& A P_{a d} A^{T}-P_{a d}-s_{n} I=0, \quad \text { for } s_{n}<0, \\
& A^{T} Q_{a d} A-Q_{a d}-r_{n} I=0, \quad \text { for } r_{n}<0 \text {. }
\end{aligned}
$$

Since

$$
\begin{gathered}
X_{I G}=U\left(S-s_{n} I\right)^{1 / 2}\left(S-s_{n} I\right)^{1 / 2} U^{T} \\
=X_{e n}-s_{n} I, \quad \text { for } s_{n}<0, \\
X_{I G}=U(S)^{1 / 2}(S)^{1 / 2} U^{T}=X_{e n}, \quad \text { for } s_{n} \geq 0, \\
Y_{I G}=V^{T}\left(R-r_{n} I\right)^{1 / 2}\left(R-r_{n} I\right)^{1 / 2} V=Y_{e n}-r_{n} I, \quad \text { for } r_{n}<0 \\
Y_{I G}=V^{T}(R)^{1 / 2}(R)^{1 / 2} V=Y_{e n}, \quad \text { for } r_{n} \geq 0 .
\end{gathered}
$$

By using Hammarling technique to calculate Cholesky factors of Gramians $P_{a d}$ and $Q_{a d}$ from realization $\left\{A, \sqrt{-S_{n}} I, \sqrt{-r_{n}} I, D\right\}$, we can write $P_{a d}=\bar{S}_{a d} \bar{S}_{a d}^{T}$ and $Q_{a d}=\bar{R}_{a d}^{T} \bar{R}_{a d}$. Therefore, frequency weighted controllability $P_{I G}$ (18) and observability $Q_{I G}$ (19) Gramians can be expressed as

$$
\begin{aligned}
P_{I G}=\bar{S}_{I G} \bar{S}_{I G}^{T}=P_{e n}+P_{a d}=S_{11} S_{11}^{T}+S_{12} S_{12}^{T}+S_{a d} S_{a d}^{T} \\
=\left[\begin{array}{lll}
S_{11} & S_{12} & S_{a d}
\end{array}\right]\left[\begin{array}{c}
S_{11}^{T} \\
S_{12}^{T} \\
S_{a d}^{T}
\end{array}\right],
\end{aligned}
$$


TABLE 1: Frequency weighted errors and error bounds comparison for reduced order models.

\begin{tabular}{ccccccccc}
\hline \multirow{2}{*}{ Weighting Order } & \multirow{2}{*}{ Enns technique [25] } & \multicolumn{2}{c}{ Campbell et al.s technique [37] } & \multicolumn{2}{c}{ Varga and Anderson's technique [38] } & \multicolumn{2}{c}{ Proposed technique } \\
& & & Error & Error bound & Error & Error bound & Error & Error bound \\
\hline \multirow{4}{*}{ Two sided } & 1 & 21.1254 & 20.5953 & 1634.2 & 21.6491 & 725.7718 & 15.6065 & 2549.0 \\
& 2 & 31.9647 & 32.8319 & 978.34 & 32.8863 & 433.8123 & 18.4571 & 1623.9 \\
& 3 & 35.0441 & 32.3860 & 590.48 & 33.9063 & 255.7017 & 26.1274 & 998.92 \\
& 4 & 28.7611 & 31.4710 & 236.41 & 30.4331 & 102.7518 & 30.7929 & 427.13 \\
\hline \multirow{4}{*}{ Input } & 5 & 12.7538 & 29.5760 & 117.81 & 12.7660 & 50.4647 & 25.6547 & 203.36 \\
\hline & 1 & 7.0257 & 7.1275 & 145.811 & 7.2356 & 92.6748 & 7.0140 & 242.5983 \\
& 2 & 10.4643 & 10.7354 & 87.9789 & 10.7694 & 55.8603 & 10.6714 & 149.7017 \\
& 3 & 11.2055 & 10.3852 & 53.0816 & 10.8477 & 32.9346 & 9.9857 & 90.8656 \\
& 4 & 8.9654 & 10.0342 & 21.8067 & 9.6079 & 13.5877 & 8.4277 & 41.1182 \\
\hline
\end{tabular}

$$
\begin{aligned}
Q_{I G} & =\bar{R}_{I G}^{T} \bar{R}_{I G}=Q_{e n}+Q_{a d}=R_{22}^{T} R_{22}+R_{12}^{T} R_{12}+R_{a d}^{T} R_{a d} \\
& =\left[\begin{array}{lll}
R_{22}^{T} & R_{12}^{T} & R_{a d}^{T}
\end{array}\right]\left[\begin{array}{l}
R_{22} \\
R_{12} \\
R_{a d}
\end{array}\right] .
\end{aligned}
$$

Remark 9. Note that, Cholesky factors for Enns and proposed technique are computed directly from augmented system realization using Hammarling technique without calculating augmented system realization Gramian matrices $P_{b i}$ and $Q_{b o}$.

\section{Illustrative Examples}

In this section, using numerical illustrative examples we show the usefulness of the proposed technique in comparison with existing frequency weighted balanced model reduction techniques for discrete time systems. Note that, proposed work deals with frequency weighted model reduction problem for discrete time systems, therefore, comparison is done with existing frequency weighted balanced model reduction techniques only.

Example 1. Consider (example C appeared in [26]) a 4th order stable discrete time system

$$
H(z)=\frac{z^{3}}{z^{4}+1.1 z^{3}-0.01 z^{2}-0.275 z-0.06}
$$

with the following weightings

$$
V_{i}(z)=W_{o}(z)=\frac{z+0.9}{z+0.1} .
$$

The first order reduced model obtained by Enns [25] technique is unstable while reduced order model obtained by Campbell et al.s, Varga and Anderson's, and proposed techniques is stable yielding frequency response errors 112.9338, 100.8739 , and 94.116 , respectively. Note that, proposed technique provides stability with relatively lower error when compared to other techniques.
Example 2. Consider a 6th order stable low pass digital elliptic filter with $0.2 \mathrm{~dB}$ of peak-to-peak ripple and a minimum stopband attenuation of $20 \mathrm{~dB}$ represented by

$$
\begin{aligned}
H(z)=( & 0.1054 z^{6}-0.1944 z^{5}+0.1187 z^{4} \\
& \left.-0.1187 z^{2}+0.1944 z-0.1054\right) \\
\times & \left(z^{6}-2.9621 z^{5}+4.8325 z^{4}-4.9819 z^{3}\right. \\
& \left.\quad+3.5245 z^{2}-1.5262 z+0.3657\right)^{-1}
\end{aligned}
$$

with the following input and output weightings, respectively,

$$
\begin{aligned}
V_{i}(z) & =\frac{z^{3}+3.0081 z^{2}+1.9944 z+1.0325}{z^{3}+0.2 z^{2}+0.75 z+0.2} \\
W_{o}(z) & =\frac{z^{3}+2.97 z^{2}+2.9403 z+0.9703}{z^{3}+1.1619 z^{2}+0.6959 z+0.1378} .
\end{aligned}
$$

Table 1 gives the comparison of error and error bounds for reduced order systems obtained by Enns, Campbell et al.s, Varga and Anderson's, and proposed techniques for the input and two sided weighting cases. Note that, the proposed technique mostly yields lower error as compared to other techniques.

Example 3. Consider a 4th order stable discrete time system [42]

$$
H(z)=\frac{10^{-3}\left(3.315 z^{3}-4.9695 z^{2}+2.1668 z-0.24002\right)}{z^{4}-3.7035 z^{3}+5.1957 z^{2}-3.2718 z+0.77986}
$$

with the following input weighting:

$$
V_{i}(z)=\frac{z^{2}-0.1 z-0.05}{z^{2}-0.9 z+0.75}
$$

Table 2 gives the comparison of error and error bounds for reduced order systems obtained by Enns, Campbell et al's Varga and Anderson's and proposed techniques for the input weighting case. Note that, the proposed technique compares well and yields relatively lower error as compared to other techniques. 
TABLE 2: Frequency weighted errors and error bounds comparison for reduced order models.

\begin{tabular}{ccccccccc}
\hline \multirow{2}{*}{ Weighting Order } & \multirow{2}{*}{ Enns technique [25] } & \multicolumn{2}{c}{ Campbell et al's technique [37] } & \multicolumn{3}{c}{ Varga and Anderson's technique [38] } & \multicolumn{2}{c}{ Proposed technique } \\
& & & Error & Error bound & Error & Error bound & Error & Error bound \\
\hline \multirow{3}{*}{ Input } & 1 & 0.0216 & 0.0245 & 0.6024 & 0.0241 & 0.3321 & 0.0240 & 0.6783 \\
& 2 & 0.0021 & 0.0027 & 0.2582 & 0.0026 & 0.1403 & 0.0025 & 0.2853 \\
& 3 & 0.0015 & 0.0025 & 0.0453 & 0.0023 & 0.0247 & 0.0021 & 0.0508 \\
\hline
\end{tabular}

TABLE 3: Frequency weighted errors and error bounds comparison for reduced order models.

\begin{tabular}{ccccccccc}
\hline \multirow{2}{*}{ Weighting Order } & \multirow{2}{*}{ Enns technique [25] } & \multicolumn{2}{c}{ Campbell et al's technique [37] } & \multicolumn{3}{c}{ Varga and Anderson's technique [38] } & \multicolumn{2}{c}{ Proposed technique } \\
& & & Error & Error bound & Error & Error bound & Error & Error bound \\
\hline \multirow{3}{*}{ Output } & 1 & 1.7905 & 1.7898 & 8.2727 & 1.7900 & 5.6369 & 1.7867 & 6.2509 \\
& 2 & 0.8967 & 0.8126 & 2.9832 & 0.8520 & 1.9243 & 0.7296 & 2.5083 \\
& 3 & 0.5098 & 0.4868 & 1.4488 & 0.4979 & 0.9219 & 0.4740 & 0.9543 \\
\hline
\end{tabular}

Example 4. Consider a 4th order stable low pass digital Chebychev type 1 filter with $0.1 \mathrm{~dB}$ of peak-to-peak ripples in the passband represented by

$$
H(z)=\frac{0.49 z^{4}-0.9799 z^{2}+0.49}{z^{4}-0.2893 z^{3}-0.6629 z^{2}+0.0246 z+0.2904}
$$

with the following output weighting:

$$
W_{o}(z)=\frac{z-0.2}{z^{2}-0.4 z+0.5} .
$$

Table 3 gives the comparison of error and error bounds for reduced order systems obtained by Enns, Campbell et al.s Varga and Anderson's and proposed techniques for output weighting. Note that, the proposed technique compares well and yields relatively lower error as compared to other techniques.

\section{Conclusion}

A new frequency weighted technique for model reduction of discrete time systems is explored. The reduced order models obtained in the presence of input, output, and two sided weightings are stable. A comparison with existing schemes shows that proposed technique provides comparable results (mostly produces lower error) for reduced order models.

\section{Conflict of Interests}

The authors declare that there is no conflict of interests.

\section{References}

[1] K. Zhou, J. C. Doyle, and K. Glover, Robust and Optimal Control, Prentice Hall, New York, NY, USA, 1996.

[2] S. Gugercin and A. C. Antoulas, "A survey of model reduction by balanced truncation and some new results," International Journal of Control, vol. 77, no. 8, pp. 748-766, 2004.

[3] A. C. Antoulas, Approximation of Large-Scale Dynamical Systems, vol. 6, SIAM, Philadelphia, Pa, USA, 2005.
[4] A. Ghafoor and V. Sreeram, "A survey/review of frequencyweighted balanced model reduction techniques," Journal of Dynamic Systems, Measurement and Control, vol. 130, no. 6, Article ID 061004, 16 pages, 2008.

[5] P. Benner, S. Gugercin, and K. Willcox, "A survey of model reduction methods for parametric systems," Tech. Rep. MPIMD/13-14, Max Planck Institute Magdeburg Preprint, Magdeburg, Germany, 2013.

[6] B. C. Moore, "Principal component analysis in linear systems: controllability, observability, and model reduction," IEEE Transactions on Automatic Control, vol. 26, no. 1, pp. 17-32, 1981.

[7] K. Glover, "All optimal Hankel-norm approximations of linear multivariable systems and their $L_{\infty}$-error bounds," International Journal of Control, vol. 39, no. 6, pp. 1115-1193, 1984.

[8] Z. Bai and R. W. Freund, "A partial Padé-via-Lanczos method for reduced-order modeling," Linear Algebra and Its Applications, vol. 332-334, pp. 139-164, 2001.

[9] S. Gugercin, "An iterative SVD-Krylov based method for model reduction of large-scale dynamical systems," Linear Algebra and Its Applications, vol. 428, no. 8-9, pp. 1964-1986, 2008.

[10] P. Benner, T. Breite, and T. Damm, "Krylov subspace methods for model order reduction of bilinear discrete-time control systems," Proceedings in Applied Mathematcs and Mechanics, vol. 1, no. 10, pp. 601-602, 2010.

[11] S. Boyd, L. El Ghaoui, E. Feron, and V. Balakrishnan, Linear Matrix Inequalities in System and Control Theory, SIAM, Philadelphia, Pa, USA, 1994.

[12] P. Gahinet and P. Apkarian, "Linear matrix inequality approach to $H_{\infty}$ control," International Journal of Robust and Nonlinear Control, vol. 4, no. 4, pp. 421-448, 1994.

[13] T. Bechtold, E. B. Rudnyi, and J. G. Korvink, Fast Simulation of Electro-Thermal MEMS, Springer, Berlin, Germany, 2007.

[14] D. K. Kumar, S. K. Nagar, and J. P. Tiwari, "Model order reduction of interval systems using mihailov criterion and factor division method," International Journal of Computer Applications, vol. 28, no. 11, 2011.

[15] B. Besselink, U. Tabak, A. Lutowska et al., "A comparison of model reduction techniques from structural dynamics, numerical mathematics and systems and control," Journal of Sound and Vibration, vol. 332, no. 19, pp. 4403-4422, 2013.

[16] C. Y. L. Dick, Multiplicative $H_{\infty}$ model reduction and fixed order output feedback controller [thesis], University of Hong Kong, Hong Kong, 2009. 
[17] H. Gao, J. Lam, C. Wang, and S. Xu, " $H_{\infty}$ model reduction for discrete time-delay systems: delay-independent and dependent approaches," International Journal of Control, vol. 77, no. 4, pp. 321-335, 2004.

[18] L. Wu, P. Shi, H. Gao, and C. Wang, " $H_{\infty}$ mode reduction for two-dimensional discrete state-delayed systems," IEE Proceedings: Vision, Image and Signal Processing, vol. 153, no. 6, pp. 769784, 2006.

[19] L. Wu, X. Su, P. Shi, and J. Qiu, "Model approximation for discrete-time state-delay systems in the TS fuzzy framework," IEEE Transactions on Fuzzy Systems, vol. 19, no. 2, pp. 366-378, 2011.

[20] L. Wu and W. X. Zheng, "Weighted $H_{\infty}$ model reduction for linear switched systems with time-varying delay," Automatica, vol. 45, no. 1, pp. 186-193, 2009.

[21] X. Su, L. Wu, P. Shi, and Y. D. Song, " $H_{\infty}$ model reduction of Takagi-Sugeno fuzzy stochastic systems," IEEE Transactions on Systems, Man, and Cybernetics B, vol. 42, no. 6, pp. 1574-1585, 2012.

[22] K. Zuhtuogullari, N. Allahvardi, and N. Arikan, "Genetic algorithm and rough sets based hybrid approach for reduction of the input attributes in medical systems," International Journal of Innovative, Computing Information \& Control, vol. 9, no. 7, pp. 3015-3037, 2013.

[23] N. H. T. Thu and Q. N. Huu, "A supervised learning method combine with dimensionality reduction in Vietnamese text summarization," International Journal of Innovative, Computing Information \& Control, vol. 9, no. 12, pp. 4903-4915, 2013.

[24] B. D. O. Anderson and Y. Liu, "Controller reduction: concepts and approaches," IEEE Transactions on Automatic Control, vol. 34, no. 8, pp. 802-812, 1989.

[25] D. F. Enns, "Model reduction with balanced realizations: an error bound and a frequency weighted generalization," in Proceedings of the 23rd IEEE Conference on Decision and Control, vol. 23, pp. 127-132, Las Vegas, Nev, USA, 1984.

[26] V. Sreeram, B. D. O. Anderson, and A. G. Madievski, "New results on frequency weighted balanced reduction technique," in Proceedings of the American Control Conference, vol. 6, pp. 4004-4009, Seattle, Wash, USA, June 1995.

[27] C.-A. Lin and T.-Y. Chiu, "Model reduction via frequency weighted balanced realization," Control Theory and Advanced Technology, vol. 8, no. 2, pp. 341-351, 1992.

[28] G. Wang, V. Sreeram, and W. Q. Liu, "A new frequencyweighted balanced truncation method and an error bound," IEEE Transactions on Automatic Control, vol. 44, no. 9, pp. 1734$1737,1999$.

[29] A. Varga and B. D. O. Anderson, "Accuracy-enhancing methods for balancing-related frequency-weighted model and controller reduction," Automatica, vol. 39, no. 5, pp. 919-927, 2003.

[30] A. Ghafoor and V. Sreeram, "Partial-fraction expansion based frequency weighted model reduction technique with error bounds," IEEE Transactions on Automatic Control, vol. 52, no. 10, pp. 1942-1948, 2007.

[31] V. Sreeram and S. Sahlan, "Improved results on frequencyweighted balanced truncation and error bounds," International Journal of Robust and Nonlinear Control, vol. 22, no. 11, pp. 11951211, 2012.

[32] U. M. Al-Saggaf and G. F. Franklin, "Model reduction via balanced realizations: an extension and frequency weighting techniques," IEEE Transactions on Automatic Control, vol. 33, no. 7, pp. 687-692, 1988.
[33] G. A. Latham and B. D. O. Anderson, "Frequency-weighted optimal Hankel-norm approximation of stable transfer functions," Systems and Control Letters, vol. 5, no. 4, pp. 229-236, 1986.

[34] Y. S. Hung and K. Glover, "Optimal Hankel-norm approximation of stable systems with first-order stable weighting functions," Systems and Control Letters, vol. 7, no. 3, pp. 165-172, 1986.

[35] V. Sreeram, S. Sahlan, W. M. W. Muda, T. Fernando, and H. H. C. $\mathrm{Iu}$, "A generalised partial-fraction-expansion based frequency weighted balanced truncation technique," International Journal of Control, vol. 86, no. 5, pp. 833-843, 2013.

[36] S. Sahlan, R. A. Rahim, and V. Sreeram, "New results of frequency weighted balanced truncation technique on Schur decomposition," in Proceedings of the 6th IEEE Conference on Industrial Electronics and Applications (ICIEA '11), pp. 113-117, Beijing, China, June 2011.

[37] K. Campbell, V. Sreeram, and G. Wang, "A Frequency-weighted discrete system balanced truncation method and an error bound," in Proceedings of the American Control Conference, vol. 4, pp. 2403-2404, Chicago, Ill, USA, June 2000.

[38] A. Varga and B. D. O. Anderson, "Accuracy enhancing methods for the frequency-weighted balancing related model reduction," in Proceedings of the 40th IEEE Conference on Decision and Control (CDC '01), vol. 4, pp. 3659-3664, Orlando, Fla, USA, December 2001.

[39] U. M. Al-Saggaf and G. F. Franklin, "An error bound for a discrete reduced order model of a linear multivariable system," IEEE Transactions on Automatic Control, vol. 32, no. 9, pp. 815819, 1987.

[40] S. Sahlan, Frequency weighted model order reduction techniques with error bounds [Ph.D. thesis], University of Western Australia, Perth, Australia, 2009.

[41] S. J. Hammarling, "Numerical solution of the stable, nonnegative definite Lyapunov equation," IMA Journal of Numerical Analysis, vol. 2, no. 3, pp. 303-323, 1982.

[42] A. Ghafoor and V. Sreeram, "Model reduction via limited frequency interval gramians," IEEE Transactions on Circuits and Systems I, vol. 55, no. 9, pp. 2806-2812, 2008. 


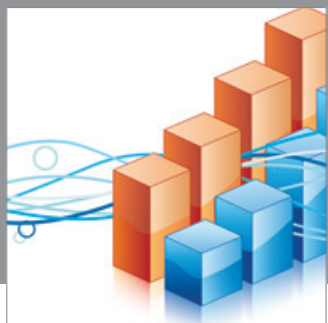

Advances in

Operations Research

mansans

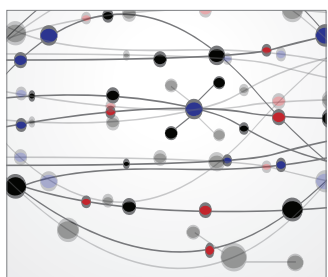

The Scientific World Journal
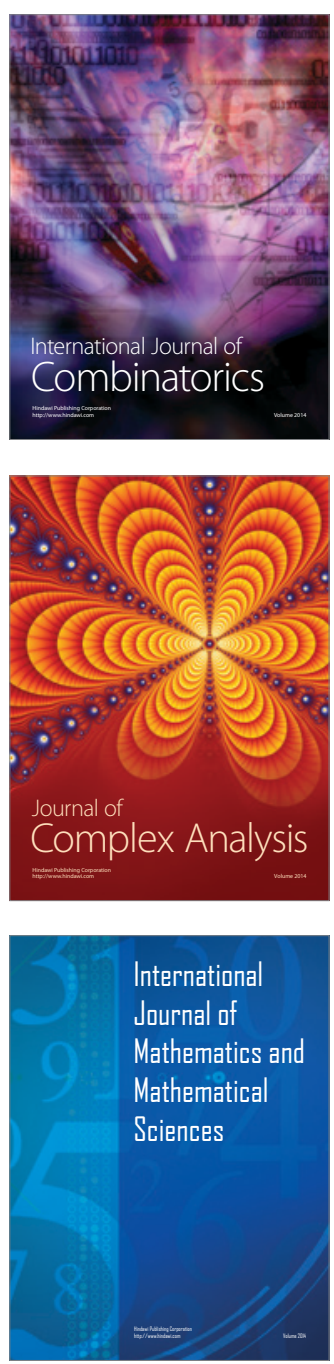
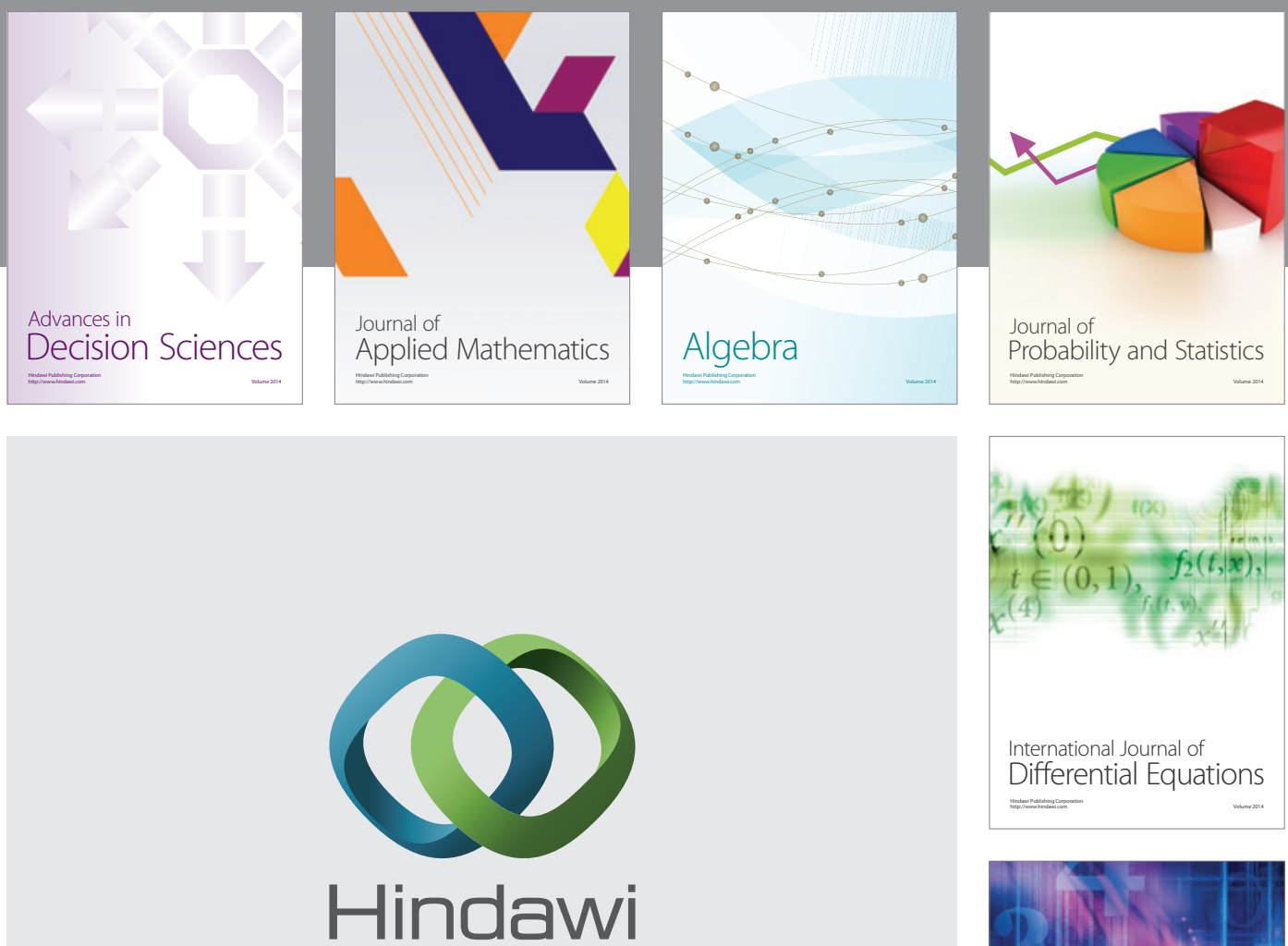

Submit your manuscripts at http://www.hindawi.com
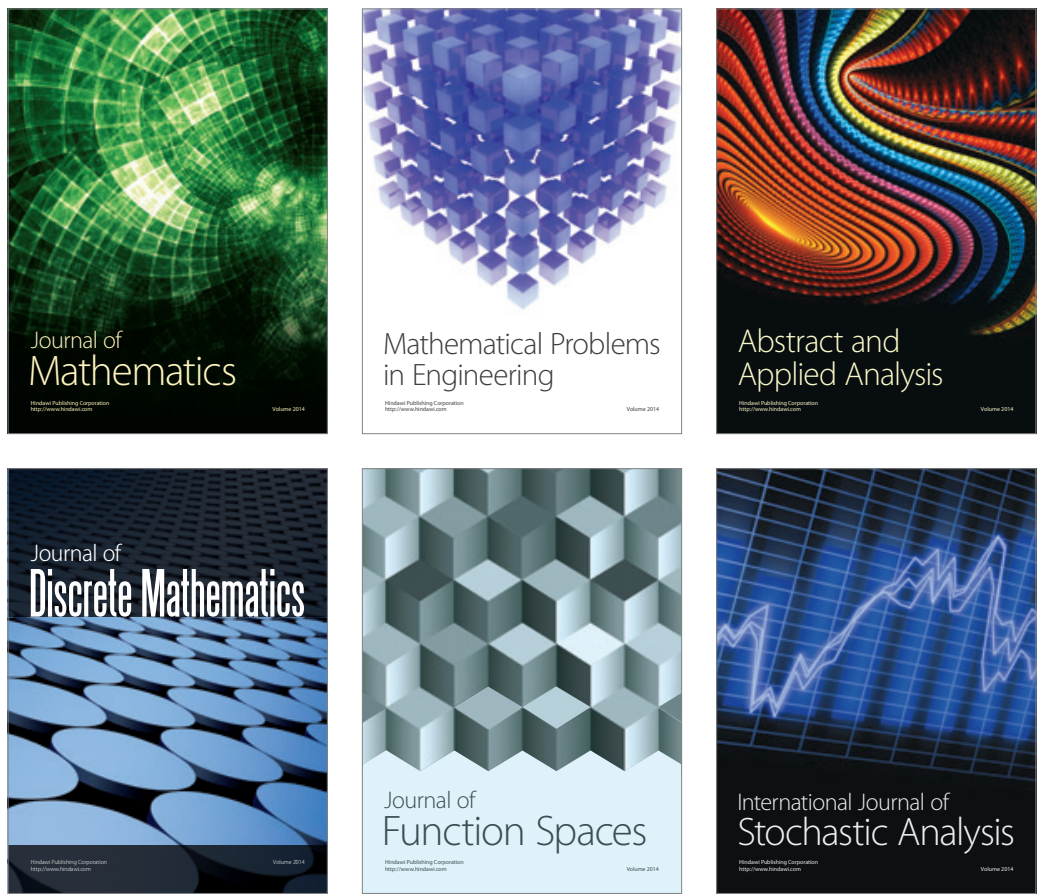

Journal of

Function Spaces

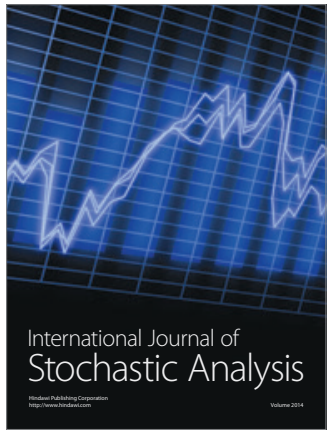

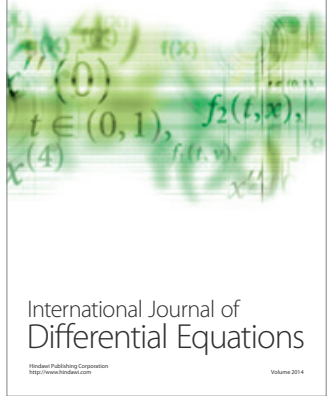
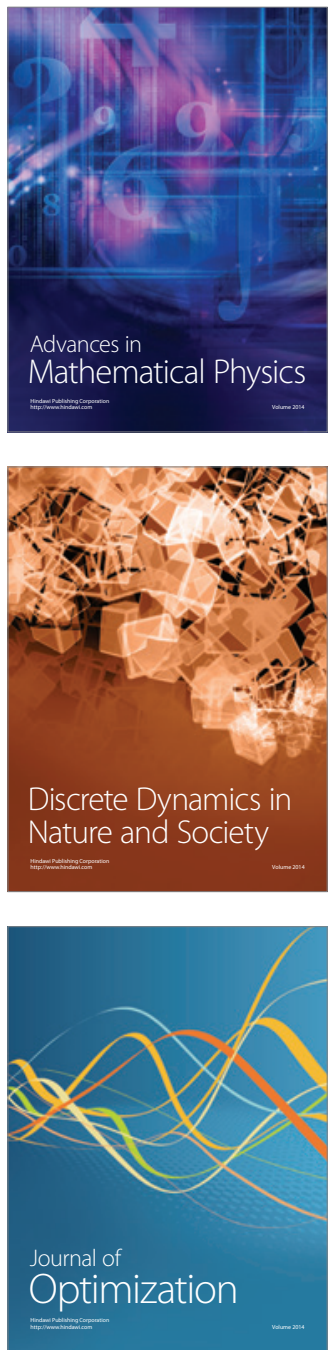